\title{
Alzheimer's Amyloid-Beta Intermediates Generated by Polymer-Nanodiscs
}

\author{
Bikash R. Sahoo , Takuya Genjo ${ }^{a}$, Michael Bekier II ${ }^{b}$, Sarah J. Coxa, Andrea K. \\ Stoddard ${ }^{a}$, Magdalena Ivanova ${ }^{a}$, Kazuma Yasuharac ${ }^{c}$, Carol A. Fierke ${ }^{\mathrm{a}, \mathrm{d}}$, Yanzhuang \\ Wang ${ }^{\mathrm{b}}$, Ayyalusamy Ramamoorthy ${ }^{* a}$
}

\author{
a.Biophysics and Department of Chemistry, University of Michigan, Ann Arbor, MI 48109-1055, USA. \\ E-mail: ramamoor@umich.edu \\ b. Department of Neurology, Molecular, Cellular and Developmental Biology, University of Michigan, \\ Ann Arbor, MI 48109-1055, USA. \\ c.Graduate School of Materials Science, Nara Institute of Science and Technology, Ikoma, Nara \\ 6300192, Japan. \\ d.Texas A\&M University, College Station, TX 77843, USA
}

\begin{abstract}
Polymethacrylate-copolymer (PMA) encased lipidnanodiscs ( $10 \mathrm{~nm})$ and macro-nanodiscs (>15 $\mathrm{nm})$ are used to study $A \beta_{1-40}$ aggregation. We demonstrate that PMAnanodiscs form a ternary association with $A \beta$ and regulate its aggregation kinetics by trapping intermediates. Results demonstrating reduced neurotoxicity of nanodisc-bound $A \beta$ oligomers are also reported.
\end{abstract}

Among various proposed causes for the onset of Alzheimer's disease $(A D),{ }^{1,2}$ the deposition of amyloid- $\beta(A \beta)$ peptides, that are sequentially cleaved from an amyloid precursor protein in the brain, has remained as the fundamental hallmark for pathogenesis. The structural plasticity of unfolded $A \beta$ monomers to adopt transient oligomers has been investigated to confer their neurotoxicity in vitro. ${ }^{3,4}$ The cell membrane has been shown to play crucial roles in modulating $A \beta$ aggregation and redirecting aggregates to several intermediate states. ${ }^{5,6,7}$ Recent studies have further shown gangliosides (GM), sphingomyelin (SM) and cholesterol to be the major modulators of $A \beta$ oligomerization. ${ }^{8,9}$ Although, recent studies proposed the catalytic activities of lipidmembrane on $A \beta$ deposition and aggregation, the exact function of these lipids is not yet fully understood. ${ }^{9,10}$ Herein, we have investigated the roles of membrane composition in modulating $A \beta$ 's aggregation using polymethacrylate (PMA) copolymer encased lipid-nanodiscs in an attempt to trap and characterize structure and toxicity of $A \beta$ intermediates.
The formation of nanodiscs with different lipid composition was achieved by varying the lipid:PMA molar ratio (see methods in the supporting information). Dynamic light scattering (DLS) measurements depicted a hydrodynamic diameter ranging from $\sim 8$ to $10 \mathrm{~nm}$ for the targeted nanodiscs (Fig.S1a). For a comparative analysis and as per the suitability of PMA to form nanodiscs, we used 1,2-dimyristoyl-snglycero-3 lipids (broad phase transition temperature suitable for biophysical studies like NMR). ${ }^{11}$ The formation of mixed lipid-nanodiscs was further validated using ${ }^{31} \mathrm{P}$ NMR experiments (Fig.S2a). We first observed the effects of PMA lipid-nanodiscs on $A \beta_{1-40}$ aggregation kinetics by the thioflavin

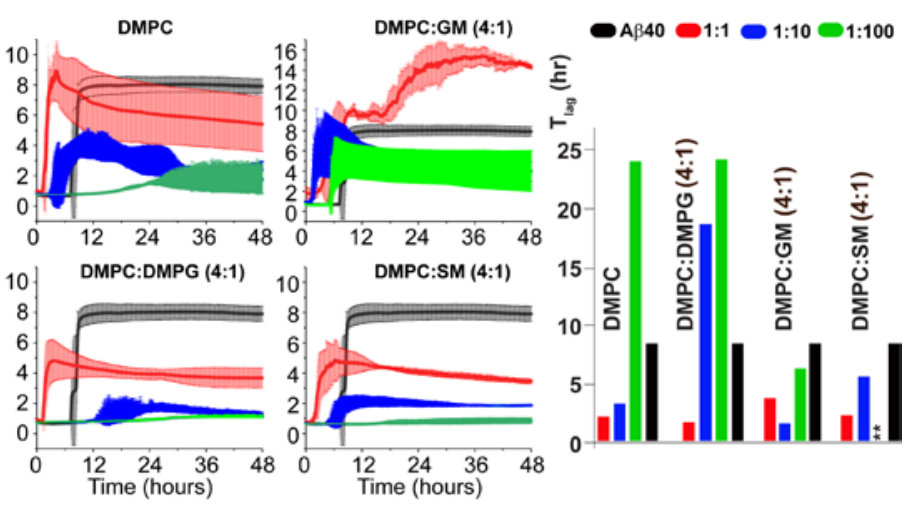

Figure 1. Aggregation kinetics of $A \beta_{1-40}$ in presence of polymer lipidnanodiscs. The aggregation kinetic profiles of $A \beta_{1-40}(5 \mu \mathrm{M})$ was monitored by the changes in ThT fluorescence as a function of time to represent peptide alone (grey trace) and in the presence of varying concentrations (color traces for the indicated $\mathrm{P}: \mathrm{L}$ ratios) of lipid-nanodiscs as shown on the top. GM, SM, DMPC and DMPG denote ganglioside, sphingomyelin, 1,2dimyristoyl-sn-glycero-3-phosphocholine and 1,2-dimyristoyl-sn-glycero-3phosphoryl-3'-rac-glycerol, respectively. The lag-time $\left(T_{\text {lag }}\right)$ is shown on the right. The asterisks indicate data not determined. 
$\mathrm{T}$ (ThT) fluorescence assay (Fig.1). Results showed both lipid concentration and composition are important to regulate $A \beta_{1}$ ${ }_{40}$ fibrillation. All nanodiscs showed no ThT binding in absence of $A \beta_{1-40}$ in solution (Fig.S1b). Although the effect of nanodiscs on $A \beta$ 's aggregation kinetics is complex (Fig.1), several trends can be observed. At 1:1 peptide:lipid molar concentration, all nanodiscs promoted aggregation irrespective of the lipid composition (Fig.1) with a significant decrease in the lag time ( $T_{\text {lag }}$.
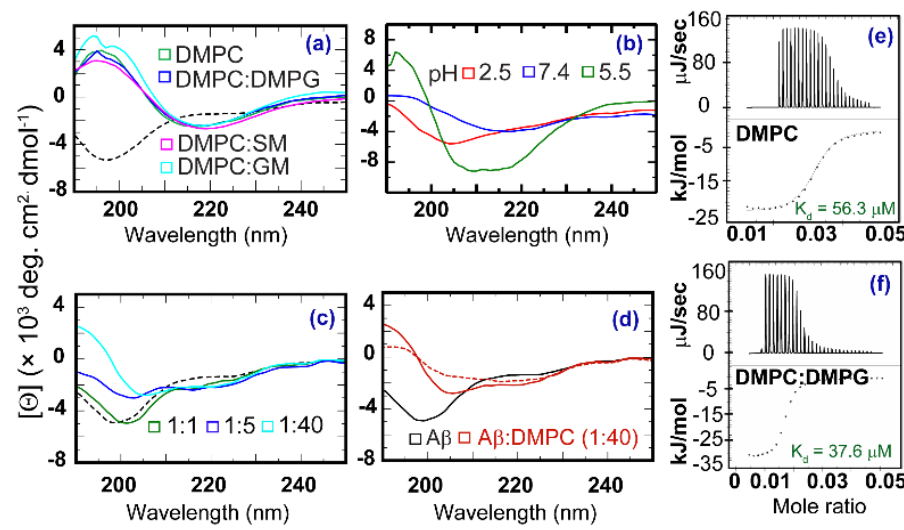

Figure 2. Conformational and kinetic analysis of $A \beta_{1-40}$ binding to nanodiscs. (a) CD spectra of $A \beta_{1-40}(25 \mu \mathrm{M})$ alone (dashed, black) and in the presence of different lipid-nanodiscs at peptide to lipid molar ratio of 1:10 (colored traces) measured at $25{ }^{\circ} \mathrm{C}$. (b) $\mathrm{pH}$ dependent conformational changes in $A \beta_{1-40}$ interacting with only PMA (red, blue) or PMA-encased DMPC nanodiscs (green) at a peptide to PMA molar ratio of 1:1. (c) CD spectra of $A \beta_{1-40}(25 \mu \mathrm{M})$ interacting with DMPC macro-nanodiscs (diameter $>16 \mathrm{~nm}$ ) at the indicated peptide:lipid molar ratio (colored traces). (d) Secondary structure transition of $A \beta_{1-40}$ due to binding to DMPC macronanodiscs as a function of time. The solid and dashed red lines denotes spectra measured at $\sim 0$ and 6 hours, respectively. (e-f) ITC thermograms showing the binding kinetics between $A \beta_{1-40}$ and lipid-nanodiscs (DMPC or 4:1 DMPC/DMPG) at a peptide $(20 \mu \mathrm{M})$ to lipid (400 $\mu \mathrm{M})$ molar ratio of 1:20.

With increasing concentration of lipids ( $P: L=1: 10)$, all nanodiscs except DMPC/DMPG (4:1) promoted $A \beta_{1-40}$ aggregation (Fig.1, red traces). Remarkably, a substantial delay in $A \beta_{1-40}$ aggregation was observed (except GM containing nanodiscs) by further increasing the lipid concentration ( $P: L=1: 100)$. The differential activity between SM and GM1 nanodiscs on modulating $A \beta_{1-40}$ aggregation kinetics are in agreement with a recent study that proposed counter protective roles of SM and $G M 1$ on $A \beta_{1-40}$ aggregation. $^{9}$

Next, we monitored the effect of varying lipid composition on the conformation of $A \beta_{1-40}$. Circular dichroism (CD) spectra of $A \beta_{1-40}$ showed a rapid ( $<5$ minutes) structural transition from a random coil with spectrum minimum at $\approx 200 \mathrm{~nm}$ to a $\beta$-sheet conformation with spectrum minimum at $\approx 216 \mathrm{~nm}$ in all nanodiscs irrespective of the lipid composition (Fig.2a).
Interestingly, a previously reported $A \beta_{1-40}$ oligomer (dimer, trimer or tetramer) also exhibited $C D$ spectral with single minima between $\approx 210 \mathrm{~nm}$ and $\approx 216 \mathrm{~nm}^{12,13}$, which could indicate that the nanodiscs-bound $\beta$-sheet species could be an oligomer. However, unlike in the presence of nanodiscs, random-coil to $\beta$-sheet structural transition in solution occurs over several days. But, our ThT results show no fiber formation in $A \beta_{1-40}$ within the $C D$ measurement time i.e $<5$ minutes for any of the lipid-nanodiscs (Fig.1). Therefore, the observation in the presence of nanodiscs suggests an induction of $\beta$-sheet-rich $A \beta_{1-40}$ oligomers that are incompetent to bind ThT and unable to proceed the selfseeding reaction as seen in the presence of high lipid concentration. The fast aggregation observed at low $\mathrm{P}: \mathrm{L}$ ratio may be attributed to the interaction of the peptide with the positively-charged PMA polymer-belt.

To explore the effect of PMA interactions with $A \beta_{1-40}$ on the observed conformational transition, $C D$ titration experiments were performed at varying $\mathrm{pH}$. Conformational changes due to $A \beta_{1-40}$ binding with PMA alone or with PMADMPC nanodiscs were measured at both acidic and basic conditions. We observed PMA at equimolar concentration of peptide in absence of lipids induced $\beta$-sheet conformation in $\mathrm{A} \beta_{1-40}$ at $\mathrm{pH} 7.4$ where it carries a net charge of -2.9 (Fig.2b, blue trace). A decrease in the buffer $\mathrm{pH}$ from 7.4 to 2.5 reduces the electrostatic interaction between PMA and $A \beta_{1-40}$ (net charge +6.7) resulting in a random-coil like $A \beta_{1-40}$ conformation (Fig. $2 b$, red trace). On the other hand, we observed a helical conformation of $A \beta_{1-40}$ mixed with DMPC nanodiscs at $\mathrm{pH}=5.5$ (the stability of nanodiscs were not affected at $\mathrm{pH}$ 5.5) where it carries nearly no charge (Fig.2b, green trace). The $\mathrm{CD}$ observation suggest that at physiological $\mathrm{pH}$, the electrostatic interaction could be the driving force for the rapid structural conversion in $A \beta_{1-40}$. Combining the $C D$ and ThT results, we speculate that a concerted ternary association between the nanodisc (lipid and PMA-belt) and the peptide modulates $A \beta$ 's folding and aggregation and could be a useful tool to trap intermediate structures as we recently observed in peptide-based lipid-nanodiscs ${ }^{14}$.

The ternary association of PMA-nanodiscs with $A \beta_{1-40}$ limits the interpretation of lipid directed $A \beta_{1-40}$ aggregation. To diminish the effect of PMA and to understand the role of lipids alone in modulating $A \beta_{1-40}$ aggregation at physiological $\mathrm{pH}$ 7.4, we designed macro-nanodiscs (diameter $>15 \mathrm{~nm}$ ) using a lipid:PMA (w/w) ratio of 4:1 (Fig.S3a). CD spectra of $A \beta_{1-40}$ in DMPC macro-nanodiscs (DMPC $C_{m d}$ ) showed a lipid concentration dependent $A \beta_{1-40}$ structural transition with a gradual change from random-coil to $\beta$-sheet conformation 
(Fig.2c). $A \beta_{1-40}$ exhibited a mixed $\alpha$-helix and $\beta$-sheet conformation for $A \beta_{1-40}: D M P C_{m d}$ molar ratio of $1: 5$ or $1: 40$ and is in agreement with previous findings. ${ }^{15}$ At molar ratios $\left(A \beta_{1}\right.$ $\left.{ }_{40}: \mathrm{DMPC}_{\mathrm{md}}\right)$ of 1:40, a time-dependent transition from $\alpha$ to $\beta$ sheet rich conformation was observed. CD spectral analysis using BeStSel ${ }^{16}$ predicted an $\alpha / \beta$ (\%) structural content of 27.2/28.5 and 8.8/20.6 in $A \beta_{1-40}$ in presence and absence of DMPC macro-nanodisc, respectively (Fig.2d). The random coil to $\alpha$-helix change in the peptide structure further ensured the lipid dependent $A \beta_{1-40}$ aggregation in macro-nanodiscs, unlike the observation from nanodiscs. The macro-nanodiscs comprising of DMPC or DMPC/SM at 4:1 molar ratio further distinguished $A \beta_{1-40}$ 's secondary structures and aggregation kinetics as compared to respective lipid-nanodiscs (Fig.S4). Taken together, these findings suggest that $A \beta_{1-40}$ aggregation in macro-nanodiscs is preferably modulated by the lipid bilayer surface as the large planar lipid bilayer enables lipidpeptide interactions with less influence of the polymer that forms the belt of the lipid-nanodisc. Thus, the studied PMAmacro-nanodiscs could be a useful tool to study membrane mediated amyloid aggregation.

Table 1. Thermodynamic parameters for $A \beta_{1-40}$ binding to PMA nanodiscs.

\begin{tabular}{lccc} 
Nanodiscs & $\boldsymbol{\Delta} \mathbf{H}$ & $\boldsymbol{\Delta S}$ & $\mathbf{T} \boldsymbol{\Delta} \mathbf{S}$ \\
\hline DMPC & -5.45 & 1.13 & 0.33 \\
DMPC:DMPG (4:1) & -6.64 & -2.02 & -0.60 \\
DMPC:DMPG (1:1) & -7.70 & -5.98 & -1.78 \\
\hline
\end{tabular}

$\Delta \mathrm{H}: \mathrm{kcal} / \mathrm{mol} ; \Delta \mathrm{S}: \mathrm{cal} / \mathrm{mol} ; \mathbf{T} \Delta \mathrm{S}: \mathrm{kcal} / \mathrm{mol} ; \mathbf{K}_{\mathrm{d}}: \mu \mathrm{M}$

We next measured the binding affinity $\left(K_{d}\right)$ of $A \beta_{1-40}$ with DMPC (100\%) or DMPC mixed with a variable amount of an anionic lipid (DMPG) nanodiscs. Remarkably, the ITC measurements showed distinct thermograms for $A \beta_{1-40}$ binding to different nanodiscs (Fig.2e and f). DMPC nanodiscs showed a micromolar $\left(K_{d}=56.3 \mu \mathrm{M}\right)$ binding affinity to $A \beta_{1-40}$ in solution (Fig.2e) which agrees well with a previous study ${ }^{5}$ that reported $K_{d}<100 \mu \mathrm{M}$ for zwitterionic liposomes. Nanodiscs containing 20 and $50 \%$ of DMPG showed different binding affinities of $37.6 \mu \mathrm{M}$ and $45.7 \mu \mathrm{M}$, respectively (Figs. $2 f$ and $S 5)$. The binding between $A \beta_{1-40}$ and DMPC nanodiscs was favored by both negative enthalpy $(-\Delta H)$ and positive entropy $(T \Delta S)$ contributions. On the other hand, $A \beta_{1-40}$ binding to anionic nanodiscs was only favored by $-\Delta H$ (Table 1$)$. The increase in $-\Delta \mathrm{H}$ from $-6.64 \mathrm{kcal} \mathrm{mol}^{-1}$ (4:1 DMPC:DMPG) to $7.70 \mathrm{kcal} \mathrm{mol}^{-1}$ (1:1 DMPC:DMPG) revealed a membrane dependent binding affinity for $A \beta_{1-40}$ at a defined PMA concentration. In addition, the $-\Delta S$ opposes $A \beta_{1-40}$ binding to anionic nanodiscs which further supported its membrane selective binding. Overall, the ITC results suggest that $A \beta_{1-40}$ interaction is membrane dependent although the cationic
PMA triggers a uniform rapid $\beta$-sheet conformation in $A \beta_{1-40}$ as revealed by $C D$.

To further examine the binding mechanism of $A \beta_{1-40}$ with nanodiscs and its conformational states, we measured $2 \mathrm{D}$ ${ }^{15} \mathrm{~N} /{ }^{1} \mathrm{H}$ SOFAST-HMQC NMR spectra of $A \beta_{1-40}$ at 10 and $25^{\circ} \mathrm{C}$, and assigned the peaks based on a previous study. ${ }^{17}$ Poorly dispersed ${ }^{15} \mathrm{~N} /{ }^{1} \mathrm{H}$ resonances obtained at $10{ }^{\circ} \mathrm{C}$ (Fig.3a, red) indicate a random-coil conformation which is in agreement with the $C D$ results (Fig.2a). At room temperature $\left(25^{\circ} \mathrm{C}\right.$, incubated for 30 minutes in solution), we observed a slight change in the chemical shift and an increase in the peak intensity (Fig.3b, blue) for the amyloid core residues. The missing Serine and Glycine resonances in solution at $25^{\circ} \mathrm{C}$, were observed in the presence of nanodiscs (DMPC:DMPG $=4: 1$ ) with 1:20 peptide:lipid molar ratio at $25^{\circ} \mathrm{C}$ (Fig.3b, green). These observations suggest that $A \beta_{1-40}$ bound to nanodiscs exhibits a short correlation time $\left(\tau_{c}\right)$ and exists in a low-ordered aggregation state in comparison to its metastable amyloid fiber. The increase in peak intensity observed for residues S8, G9, K16, G25, S26 and V36 indicate the induction of a more flexible structure for the selected domains (Fig.S6). In contrast, the core domain comprising of residues 17-20 show a substantial decrease in peak intensity and suggests a membrane-associated and folded state. ${ }^{7,18}$ Taken together, the ThT aggregation kinetics that showed a $T_{\text {lag }}$ over 20 hrs (Fig.1) for 4:1 DMPC:DMPG nanodiscs at 1:20 peptide:lipid molar ratio correlates with the dispersed ${ }^{15} \mathrm{~N} /{ }^{1} \mathrm{H}$ NMR resonances, unlike that observed for matured fibers (Fig.S2c). This indicates the formation of low-ordered $A \beta_{1-40}$ aggregates in presence of nanodiscs.
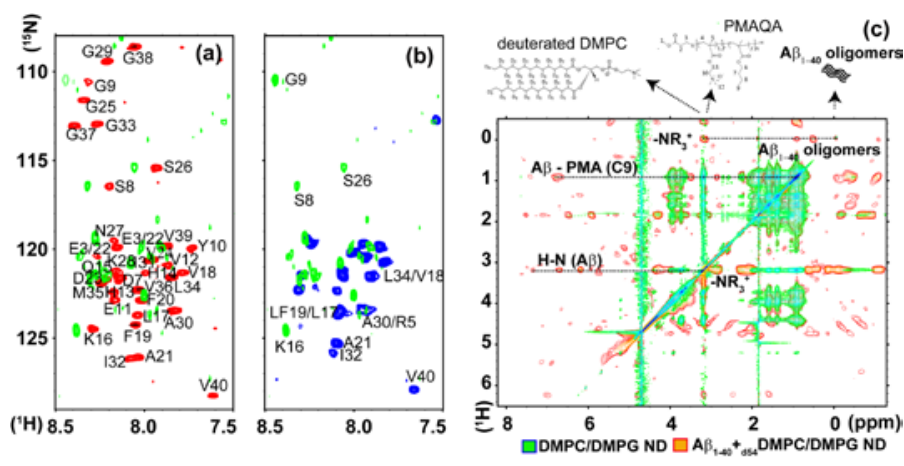

Figure 3. Structural and functional characterization of $A \beta_{1-40}$ in lipidnanodiscs. $(a-b){ }^{15} \mathrm{~N}$ SOFAST-HMQC spectra of $A \beta_{1-40}(70 \mu \mathrm{M})$ titrated with $1.4 \mathrm{mM}$ DMPC:DMPG (4:1) nanodiscs in $10 \mathrm{mM}$ sodium phosphate buffer solution (green). The red and blue spectra represent the peptide spectra acquired in absence of nanodiscs at $10{ }^{\circ} \mathrm{C}$ and $25^{\circ} \mathrm{C}$, respectively. (c) $2 \mathrm{D}$ NOESY spectra of $70 \mu \mathrm{M}$ monomeric $A \beta_{1-40}$ in presence (red-yellow) or absence (green-blue) of $1.4 \mathrm{mM}$ d54-DMPC/DMPG (4:1) nanodiscs at $25{ }^{\circ} \mathrm{C}$. The dotted (horizontal) lines show the correlation peaks between the amide-protons of $A \beta_{1-40}$ and the side chain resonances (C9 and-NR ${ }_{3}^{+}$) of the PMA-belt in nanodiscs. Chemical structures of d54-DMPC and PMA are shown at the top. NMR spectra were recorded on a $600 \mathrm{MHz}$ Bruker NMR spectrometer using a cryoprobe. 
$2 \mathrm{D}{ }^{1} \mathrm{H} /{ }^{1} \mathrm{H}$ NOESY spectrum showed two diagonal peaks close to $0 \mathrm{ppm}$ indicating the presence of $A \beta_{1-40}$ oligomers (Fig.3c). ${ }^{19}$ These oligomer peaks show cross-peaks with PMA's hydrophobic chain ( $\mathrm{C} 9$ atom) and the quaternary ammonium of polymer or lipids (Fig.3c). Both the amide ( $\mathrm{H}-\mathrm{N}$ ) and side chain protons $(\gamma)$ of $A \beta_{1-40}$ show a substantial correlation with PMA functional groups (Fig.3c). The corresponding ${ }^{1} \mathrm{H}$ NMR spectra of $A \beta_{1-40}$ (Fig.S7b) showed a substantial dispersion in the amide region that are significantly different from $A \beta_{1-40}$ monomers and fibers (Fig.S2b and c). The NOESY spectrum indicates a direct interaction between the quaternary ammonium group of PMA (in nanodiscs) and $A \beta_{1-40}$.

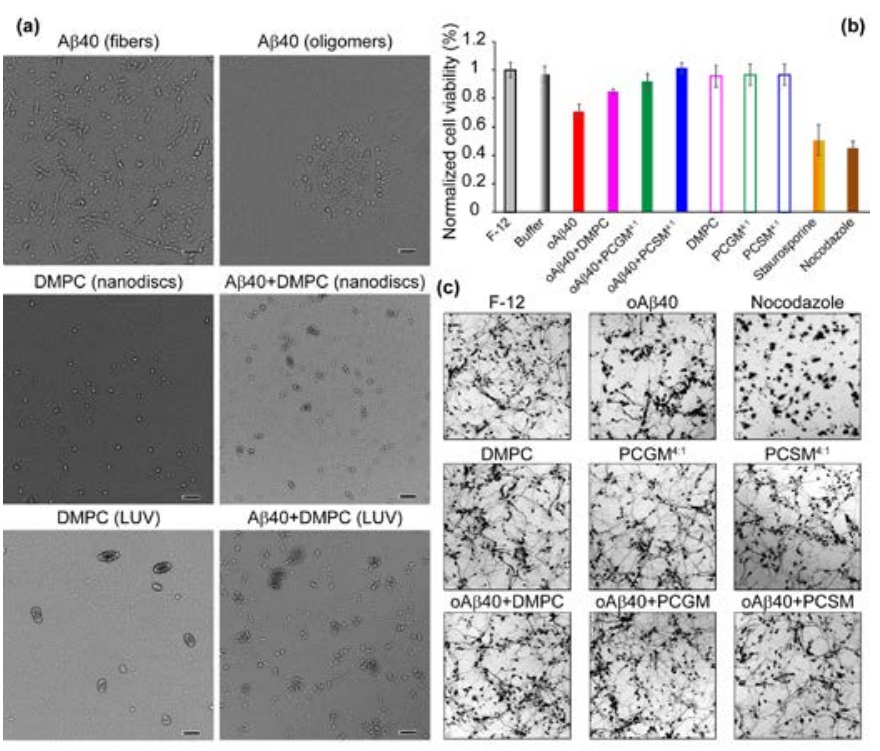

Figure 4. TEM and fluorescence images of nanodiscs and SH-SY5Y cells treated with $A \beta_{1-40}$. (a) TEM images of $A \beta_{1-40}$ fibers, oligomers and intermediates in presence of DMPC nanodiscs or LUVs measured after 48 hours. The scale bar is $200 \mathrm{~nm}$. (b) MTT assays showing the formazan absorbance expressed as a measure of cell viability from SH-SY5Y cultured cells treated with $5 \mu \mathrm{M}$ of $A \beta_{1-40}$ oligomers (oA $\beta 40$ ) in presence and absence of nanodiscs. (c) Fluorescence images of neuronal damage caused by $5 \mu \mathrm{M}$ of $A \beta_{1-40}$ oligomers in presence and absence of nanodiscs. $F-12$ is the negative control; staurosporine and nocodazole were used as positive controls.

TEM images of $A \beta_{1-40}$ fibers and oligomers, freshly prepared by dissolving monomers (see Methods in supporting information), showed morphologically distinct species after $48 \mathrm{hrs}$ (Fig.4a). No $A \beta_{1-40}$ fibers were observed in presence of DMPC nanodiscs (Fig.4a, middle). While nanodiscs fusion and aggregated spherical populations were seen in $A \beta_{1-40}$ treated DMPC-nanodiscs (Fig.4a), a mixed fibril and spherical $A \beta_{1-40}$ species were observed in presence of DMPC-LUVs (Fig.S3b). These observations demonstrate a successful trapping of lower-ordered aggregates/intermediates of $A \beta_{1-40}$ species using nanodiscs (Fig.4a).

Protein-based nanodiscs have been shown to control the aggregation and toxicity of $A \beta_{1-40}$ in-vitro and in-vivo ${ }^{14,20-22}$.
Thus, we studied the potential use of PMA-nanodiscs in modulating the toxicity of $A \beta_{1-40}$ oligomers. The $A \beta_{1-40}$ oligomers treated to human neuroblastoma (SH-SY5Y) cells were observed to be neurotoxic with a substantial reduction in cell-viability (Fig. 4b), and fluorescence imaging showed a severe damage to neurites and soma (Figs $4 c$ and S8). Whereas, the $A \beta_{1-40}$ oligomers incubated with PMA-nanodiscs exhibited relatively less neurotoxic and neuronal damage as shown in Fig.4b and c; the least toxicity observed for DMPC/SM nanodiscs is in agreement with the reported role of $\mathrm{SM}^{23}$ Morphometric analysis of neurons treated with nanodisc containing $A \beta$ intermediates showed well-connected neurites that were severely damaged by oligomers and small molecules like staurosporine and nocodazole (Figs. 4c and S8). $A \beta_{1-40}$ monomers or fibers interacting with nanodiscs showed a loss of $\approx 25 \%$ cell viability, whereas $A \beta_{1-40}$ oligomers exhibited significant $(\approx 60 \%)$ cytotoxicity (Fig.S9). Thus, we have demonstrated the PMA encased nanodiscs are potential molecules to protect neuron cells by reducing the toxicity of $\mathrm{A} \beta_{1-40}$ species.

Structural and mechanistic studies presented here revealed a symbiotic functional relationship for PMA and its associated membrane lipids. While PMA induces a rapid structural transition in $A \beta_{1-40}$, a controllable intermediate of $A \beta_{1-40}$ can be generated by varying the lipid composition and concentration in PMA-nanodiscs (Figs.1 and 2). In addition, as evidenced from NMR results that showed a dispersed ${ }^{1} \mathrm{H} /{ }^{15} \mathrm{~N}$ resonance distribution and intermolecular ${ }^{1} \mathrm{H} /{ }^{1} \mathrm{H}$ correlation, polymer-nanodiscs can be used to track amyloid intermediates for real-time measurements (Fig.3). It is also remarkable to note that the "styrene-free" PMA-nanodiscs enable fluorescence and $C D$ experiments for real-time characterization of amyloid aggregation and are useful for conformational analysis of amyloidogenic proteins embedded in a lipid bilayer.

While investigating the structure, dynamics and function of $A \beta_{1-40}$ in membrane interface remains challenging due to the complexity, we have proposed the applicability of nanodiscs and macro-nanodiscs to decipher the molecular mechanism of seeding reaction at atomistic-scale on realtime. PMA encased lipid nanodiscs generates off-pathway lower-order aggregates of $A \beta_{1-40}$ directed by PMA and modulated by lipid composition over time. Based on the NMR observation (Fig.3), we propose a distinct structural ensemble of $A \beta_{1-40}$ intermediates. The toxicity of $A \beta_{1-40}$ oligomers were substantially lowered by nanodiscs and are dependent on the lipid properties and yielded distinct morphological phenotypes as revealed from TEM and cell assay (Fig.4). ${ }^{24}$ 
In conclusion, we have successfully demonstrated a new approach to trap $A \beta_{1-40}$ intermediates and characterize them at atomic-level using polymer nanodiscs for the first time. The significant binding efficacy of PMA-nanodiscs with $A \beta_{1-40}$ species reported in this study could be valuable in the development of potential therapeutic strategies for Alzheimer's disease. While the lack of understanding and high-resolution structures of membrane-bound $A \beta_{1-40}$ intermediates have been a bottle-neck ${ }^{25}$, the structural and functional insights into $A \beta_{1-40}$ intermediates reported in this study could also be valuable for the development of compounds to suppress neuronal cell death and potentially develop treatment for Alzheimer's disease. The ability of polymer-based nanodiscs to trap amyloid intermediates and remodel them to pathologically distinct morphological states is unique and will have broad impacts in the structural characterization of a variety of membrane-assisted amyloid aggregation processes that are implicated in various neurodegenerative disorders. Further, this approach would also enable the application of a variety of physical techniques for high-throughput characterization of protein misfolding, nanodisc encapsulated $A D$ drug delivery and screening of small molecule compounds for the development of therapeutics. While the PMA-nanodiscs render a curvaturefree lipid bilayer to probe the role of the lipid membrane on amyloid aggregation, further investigation to fully understand the mechanism of the polymer-belt induced formation of $\beta$ sheet structure of $A \beta$ and the use of PMA-nanodiscs on other amyloid proteins would be useful. Further development of different types of polymer-belts like peptide and apolipoproteins-encased nanodiscs ${ }^{14,22}$ for the stabilization of amyloid oligomers would be also beneficial for high resolution structural studies.

\section{Acknowledgements}

This study was supported by NIH (AG048934 to A.R.). We thank Professor Bernd Reif for providing us the recombinant expression system and protocol for the production of amyloid-beta-1-40 peptide.

\section{Conflicts of interest}

There are no conflicts to declare.

\section{References}

1 T. P. J. Knowles, M. Vendruscolo and C. M. Dobson, Nat. Rev. Mol. Cell Biol., 2014, 15, 496-496.

2 C. L. Masters, R. Bateman, K. Blennow, C. C. Rowe, R. A. Sperling and J. L. Cummings, Nat. Rev. Dis. Prim., 2015, 1, 15056.

3 S. M. Chafekar, F. Baas and W. Scheper, Biochim. Biophys. Acta - Mol. Basis Dis., 2008, 1782, 523-531. A. Sebollela, G. M. Mustata, K. Luo, P. T. Velasco, K. L. Viola, E. N. Cline, G. S. Shekhawat, K. C. Wilcox, V. P.

Dravid and W. L. Klein, ACS Chem. Neurosci., 2014, 5, 1238-1245.

M. S. Terakawa, H. Yagi, M. Adachi, Y. H. Lee and Y. Goto, J. Biol. Chem., 2015, 290, 815-826.

S. A. Kotler, P. Walsh, J. R. Brender and A. Ramamoorthy, Chem. Soc. Rev., 2014, 43, 6692-6700. K. J. Korshavn, A. Bhunia, M. H. Lim and A. Ramamoorthy, Chem. Commun., 2016, 52, 882-885. M. Thomaier, L. Gremer, C. Dammers, J. Fabig, P. Neudecker and D. Willbold, Biochemistry, 2016, 55, 6662-6672.

M. Amaro, R. Šachl, G. Aydogan, I. I. Mikhalyov, R. Vácha and M. Hof, Angew. Chemie - Int. Ed., 2016, 55, 9411-9415.

J. Habchi, S. Chia, C. Galvagnion, T. C. T. Michaels, M. M. J. Bellaiche, F. S. Ruggeri, M. Sanguanini, I. Idini, J. R. Kumita, E. Sparr, S. Linse, C. M. Dobson, T. P. J. Knowles and M. Vendruscolo, Nat. Chem., , DOI:10.1038/s41557-018-0031-x.

K. Yasuhara, J. Arakida, T. Ravula, S. K. Ramadugu, B. Sahoo, J.-I. Kikuchi and A. Ramamoorthy, J. Am. Chem. Soc., 2017, jacs.7b10591.

S. A. Kotler, P. Walsh, J. R. Brender and A. Ramamoorthy, 2015, 43, 6692-6700.

K. Ono, M. M. Condron and D. B. Teplow, Proc. Natl. Acad. Sci., 2009, 106, 14745-14750.

B. R. Sahoo, T. Genjo, S. J. Cox, A. K. Stoddard, G. M. Anantharamaiah, C. Fierke and A. Ramamoorthy, J. Mol. Biol., DOI:10.1016/j.jmb.2018.08.018.

M. Bokvist, F. Lindström, A. Watts and G. Gröbner, J. Mol. Biol., 2004, 335, 1039-1049.

A. Micsonai, F. Wien, L. Kernya, Y.-H. Lee, Y. Goto, M. Réfrégiers and J. Kardos, Proc. Natl. Acad. Sci., 2015, 112, E3095-E3103.

S. Vivekanandan, J. R. Brender, S. Y. Lee and A. Ramamoorthy, Biochem. Biophys. Res. Commun., 2011, 411, 312-316.

Y. Shafrir, S. Durell, N. Arispe and H. R. Guy, Proteins Struct. Funct. Bioinforma., 2010, 78, 3473-3487.

S. Narayanan and B. Reif, Biochemistry, 2005, 44, 14441452.

Q. Song, H. Song, J. Xu, J. Huang, M. Hu, X. Gu, J. Chen, G. Zheng, H. Chen and X. Gao, Mol. Pharm., 2016, 13, 3976-3987.

H. Song, X. Ma, J. Xu, Q. Song, M. Hu, X. Gu, Q. Zhang, L. Hou, L. Chen, Y. Huang, P. Yu, D. Wang, G. Jiang, M. Huang, J. Chen, H. Chen and X. Gao, Nano Res., 2018, 114.

Q. Song, M. Huang, L. Yao, X. Wang, X. Gu, J. Chen, J. Chen, J. Huang, Q. Hu, T. Kang, Z. Rong, H. Qi, G. Zheng, H. Chen and X. Gao, ACS Nano, 2014, 8, 2345-2359. N. J. Haughey, V. V. R. Bandaru, M. Bae and M. P. Mattson, Biochim. Biophys. Acta-Mol. Cell Biol. Lipids, 2010, 1801, 878-886.

A. Piccini, R. Borghi, M. Guglielmotto, E. Tamagno, G. Cirmena, A. Garuti, V. Pollero, S. Cammarata, M. Fornaro, M. Messa, L. Colombo, M. Salmona, G. Perry and M. Tabaton, J. Neurochem., 2012, 122, 1023-1031. C. Haass and D. J. Selkoe, Nat. Rev. Mol. Cell Biol., 2007, 8, 101-112. 